\title{
Implementation of a Web-Based Work-Related Psychological Aftercare Program Into Clinical Routine: Results of a Longitudinal Observational Study
}

Rüdiger Zwerenz ${ }^{1}$, Dr Biol Hom; Carlotta Baumgarten ${ }^{1}, \mathrm{MSc}$; Ingo Dahn², Dr Sc; Nicole Labitzke ${ }^{3}$, PhD; Andreas Schwarting ${ }^{4}$, Dr Med; Matthias Rudolph ${ }^{5}$, Dr Med; Peter Ferdinand ${ }^{2}$, PhD; Ute Dederichs-Masius ${ }^{6}$, Dr Med; Manfred E Beutel ${ }^{1}$, Dr Med

\footnotetext{
${ }^{1}$ Department for Psychosomatic Medicine and Psychotherapy, University Medical Center, Johannes Gutenberg-University, Mainz, Germany

${ }^{2}$ Knowledge Media Institute, University of Koblenz-Landau, Koblenz, Germany

${ }^{3}$ Center for Audiovisual Production, Johannes Gutenberg-University, Mainz, Germany

${ }^{4}$ Center of Rheumatology, University Medical Center, Johannes Gutenberg-University, Mainz, Germany

${ }^{5}$ Mittelrhein-Klinik, German Statutory Pension Insurance Rhineland-Palatinate, Boppard, Bad Salzig, Germany

${ }^{6}$ Drei-Burgen-Klinik, German Statutory Pension Insurance Rhineland-Palatinate, Bad Kreuznach, Germany
}

\section{Corresponding Author:}

Rüdiger Zwerenz, Dr Biol Hom

Department for Psychosomatic Medicine and Psychotherapy

University Medical Center

Johannes Gutenberg-University

Untere Zahlbacher Str 8

Mainz, 55131

Germany

Phone: 496131175981

Fax: 49613117475981

Email: ruediger.zwerenz@unimedizin-mainz.de

\section{Abstract}

Background: As inpatient medical rehabilitation serves to promote work ability, vocational reintegration is a crucial outcome. However, previous Web-based trials on coping with work-related stress have been limited to Web-based recruitment of study participants.

Objective: The aim of our study was to evaluate the implementation of an empirically supported transdiagnostic psychodynamic Web-based aftercare program GSA (Gesund und Stressfrei am Arbeitsplatz [Healthy and stress-less at the workplace])-Online plus into the clinical routine of inpatient medical rehabilitation, to identify characteristics of patients who have received the recommendation for GSA-Online plus, and to determine helpfulness of the intervention and satisfaction of the participants as well as improvement in quality of life and mental health status of the regular users of GSA-Online plus.

Methods: GSA-Online plus was prescribed by physicians at termination of orthopedic psychosomatic inpatient rehabilitation. Participants' use of the program, work-related attitudes, distress, and quality of life were assessed on the Web.

Results: In 2 rehabilitation centers, 4.4\% (112/2562) of rehabilitants got a recommendation for GSA-Online plus during inpatient rehabilitation. Compared with usual person aftercare, the Web-based aftercare program was rarely recommended by physicians. Recommendations were made more frequently in psychosomatic $(69 / 1172,5.9 \%)$ than orthopedic $(43 / 1389,3.1 \%)$ rehabilitation $\left(\chi_{1}^{2}=11.845, P=.001\right.$, Cramér $\left.V=-0.068\right)$ and to younger patients $(P=.004, d=0.28)$ with longer inpatient treatment duration $(P<.001, r=-0.12)$ and extended sick leaves before inpatient medical rehabilitation $(P=.004 ;$ Cramér V=0.072). Following recommendation, $77 \%$ (86/112) of rehabilitants participated in Web-based aftercare. Completers (50/86, 58\%) reported statistically significant improvements between discharge of inpatient treatment and the end of the aftercare program for subjective work ability $(P=.02, d=0.41)$, perceived stress $(P=.01, d=-0.38)$, functioning $(P=.002, d=-0.60)$, and life satisfaction $(P=.008, d=0.42)$. 
Conclusions: Physicians' recommendations of Web-based aftercare are well accepted by patients who derive considerable benefits from participation. However, a low rate of prescription compared with other usual aftercare options points to barriers among physicians to prescribing Web-based aftercare.

(J Med Internet Res 2019;21(6):e12285) doi: 10.2196/12285

\section{KEYWORDS}

aftercare; internet; mental health; psychotherapeutic processes; return to work; occupational stress; health plan implementation

\section{Introduction}

\section{Occupational Stress and Work-Related Medical Rehabilitation}

Work-related stress, as observed in one-third of the German population [1], is an important risk factor for common mental disorders [2]. Conversely, mental disorders have become the leading reasons for long-term sickness absence [3] and premature pension in Germany [4]. The main purpose of inpatient medical rehabilitation is to restore or promote capacity to work of rehabilitants. Work-related medical rehabilitation (WMR) focusing on the workplace situation has been implemented successfully but needs to be complemented by work-related aftercare interventions to support rehabilitants during vocational reintegration [5]. However, evidence has been mixed. First, studies with intensified work-related orthopedic rehabilitation aftercare have not proven to be effective compared to aftercare without a focus on work-related topics [6]. On the contrary, participation in a graded return to work program reduced the relative risk of permanent work disability by about $40 \%$ as well as the time of welfare benefits owing to sickness absence compared with matched controls [7]. Yet, participation in aftercare programs following inpatient medical rehabilitation is low because of long waiting times, the lack of local aftercare providers, or incompatibility with family or work commitments $[8,9]$.

Web-based interventions have been shown to be effective for a broad range of mental disorders, for example, depression [10], anxiety disorders [11], pain [12], substance abuse [13], and also improved physical activity [14] or a healthy diet and weight reduction [15] or psychosocial support for patients with chronic diseases [16]. The majority of the German working population (nearly 90\%) is on the Web using the internet daily (72\%) [17] and a substantial part is using it as a frequent source of health information (38\%) [18]. Thus, Web- and mobile-based interventions may close gaps in routine care and improve diagnostics and treatment in medical rehabilitation [19] as widely accessible and cost-effective interventions [20].

\section{Web-Based Aftercare}

Initial results of self-guided Web-based stress management interventions have been mixed. In a recent meta-analysis for Web-based interventions, moderate effect sizes were reported for overall 26 studies in reducing work-related stress $(d=0.43$, 95\% CI 0.31-0.54) [21]. An internet- and mobile-based stress management program has proven effective in reducing perceived stress ( $d=0.96$ posttreatment; $d=0.65$ 6-month follow-up) compared with a waiting list control group and improved other relevant parameters of mental health, for example, depression, anxiety, and emotional exhaustion [22,23]. However, Web-based interventions when following inpatient medical rehabilitation are still rare [24] and interventions tested in randomized controlled trials have rarely been transferred into routine care.

We developed a Web-based transdiagnostic aftercare program (GSA-Online; Gesund und Stressfrei am Arbeitsplatz [Healthy and stress-less at the workplace]) that aimed at improving vocational reintegration of rehabilitants after long-term sickness absence. In our previous randomized controlled trial, GSA-Online had a statistically significant positive influence on the subjective prognosis of gainful employment $(d=0.13$ at the end of the intervention and $d=0.20$ at the follow-up 12 months after study inclusion). Furthermore we could show positive effects on affective mental distress (eg, $d=0.25$ for generalized anxiety or $d=0.18$ for depressive symptoms at follow-up) of the rehabilitants participating in the intervention [25] compared to the participants of an active control group.

\section{Study Aims and Research Question}

The aims of this study were (1) to examine whether the further developed GSA-Online plus can be implemented into routine care of inpatient medical rehabilitation, (2) to identify characteristics of patients who have received the recommendation for GSA-Online plus, and (3) to determine perceived helpfulness of the intervention and satisfaction of the participants. With regard to our primary outcome, we hypothesized that (1) GSA-Online plus would be recommended as often as other established aftercare programs for rehabilitants with a special need for WMR-like psychological treatment, rehabilitation aftercare, or vocational rehabilitation; (2) at least $66 \%$ of the rehabilitants with a recommendation for GSA-Online plus would participate and write at least 1 diary entry after rehabilitation; and (3) completers, that is, regular users with at least 6 diary entries, would achieve (a) a more positive subjective prognosis of gainful employment, (b) an increased quality of life and lower perceived stress, and (c) an improvement of emotional distress such as depression and anxiety at the end of the intervention compared to discharge from rehabilitation.

\section{Methods}

\section{Study Design}

The study was conducted in 2 rehabilitation centers treating 3 different medical indications (psychosomatic and orthopedic rheumatic diseases). Inpatient medical rehabilitation entails a multimodal group-oriented approach, supplemented by individual therapy, addressing work ability of rehabilitants with chronic (>6 months), somatic, or psychological impairments [26]. In a pre-post design, data collection took place at 14 time 
points: after discharge from inpatient rehabilitation, that is, at the beginning (T1), once a week during (T2-T13; only active participants), and at the end of the aftercare intervention (T14).

Recruitment of the study was conducted for 10 months between June 2016 and March 2017. Inclusion criteria were (1) employment and a plan to return to work within 4 weeks after inpatient medical rehabilitation, (2) the ability to write in German language, (3) age between 18 and 59 years, and (4) a private internet access. During inpatient rehabilitation, the treating physician could prescribe the self-explanatory program by handing out an information leaflet to the patient and document it as a recommendation [27]. The recommendation was collected by the study assistant in each clinic who gave further study information, collected written consent, and gave access data for GSA-Online plus to the participant.

\section{Intervention}

The intervention GSA-Online plus aimed to support occupationally stressed rehabilitants during their return to work. In a structured psychodynamic format [28], participants were instructed to identify and articulate interpersonal and intrapsychic problems during return to work. Participants got weekly personalized writing impulses from a trained and supervised therapist to help them write in the form of a diary about their experiences of returning to their workplace. Therapeutic commentaries (usually within 24 hours) to their Web-based diary entry processed interpersonal and intrapsychic problems and helped participants deal with their individual job-related problems and stabilize their working capacity. Usually, it took 20 to 40 min per week per participant for the therapist to write an answer to the diary entry and add another writing impulse. In addition to the previous version (GSA-Online), educational video clips were used to familiarize participants with the program and its features (see Multimedia Appendices 1-4). For a detailed description of the therapeutic rationale, see Beutel et al [28].

\section{Measures}

As primary outcomes, the medical referral rate was documented (frequency of recommendations of GSA-Online plus) and participants' utilization of GSA-Online plus was tracked with PIWIK (now Matomo [29]), a secure open Web analytics platform and assessed with self-constructed single items (eg, "Please indicate how often you have used GSA-Online plus since the end of your inpatient treatment.").

In pre- and postmeasurements, the secondary outcome subjective prognosis of gainful employment was assessed with the SPE-Scale [30] consisting of 3 items assessing a subjective rating of future employment until retirement age, the impairment of work ability by the current health status, as well as the plan to apply for a premature pension. The 3 items could be added up to a score between 0 and 3 with a higher score indicating a higher risk for premature pension. The capacity to work was assessed with the short form of the work ability index (WAI), a 7-item scale with a reliability of alpha $=.78$ in a German population (eg, "How do you estimate your current work ability in terms of physical work requirements?") [31]. Mental disorders were assessed with different subscales of the German version of the Patient Health Questionnaire (PHQ-D) by Löwe et al [32]. Depressive symptoms were assessed with the 9-item scale PHQ-9 (eg, "Over the last two weeks, how often have you been bothered by little interest and pleasure in doing things?") [33], with an internal consistency of alpha=.89. Stress symptoms were assessed with the 10-item stress module PHQ-Stress (eg, "Over the last four weeks, how often have you been bothered by worries about health?” ) [34,35]. Anxiety symptoms were assessed with the 7-item scale for General Anxiety Disorder (GAD-7; eg, "Over the last 2 weeks, how often have you been bothered by feeling nervous, anxious or on edge?", alpha=.92) [36]. Somatoform symptoms were assessed with the 8 items of the Somatic Symptom Scale-8 (eg, "During the past 7 days, how much have you been bothered by stomach or bowel problems?", alpha=.81) [37] also based on the PHQ. Psychosocial stressors were measured with the 4-item short form of the Perceived Stress Scale (PSS-4; eg, "In the last month how often have you felt you were unable to control the important things in your life?", alpha=.60-.82) [38]. General functioning was measured with the 3-item Sheehan Disability Scale (eg, "To what extent do your symptoms impair your functioning in your social life?", alpha=.89) [39,40], where each item can be scored from 0 to 10 resulting in a global score from 0 (unimpaired) to 30 (highly impaired). Resources were assessed with the 3-item Oslo Social Support Scale (eg, "How many people are so close to you that you can count on them if you have great personal problems?", alpha=.60) [41], with the 4-item Brief Resilient Coping Scale (eg, "I look for creative ways to alter difficult situations", internal consistency $r=0.76$ ) [42], with the Loneliness Scale [43] and with the questions on life satisfaction, a scale that consists of 28 -item modules (general life satisfaction and satisfaction with health, alpha=.82-89) [44]. Patient satisfaction was assessed with the 8-item Client Satisfaction Questionnaire (eg, "How would you rate the quality of the service you have received?", alpha=.93) [45]. In a weekly query, participants were asked with 2 items on a 5-point Likert scale (from $0=$ not at all to $4=$ very) about their satisfaction with GSA-Online plus ("How satisfied are you with the feedback of the online therapist?" and "How helpful was the feedback of the online therapist?") and rated their overall health condition with 1 item of the German version of the EuroQol Questionnaire (EQ-5D) [46] ("Your own health status today") as well as their current work ability with the first item from the WAI [31] also known as the Work Ability Score (WAS) [47], each on a Likert scale from 0 to 10 . At the end of the aftercare program, participants were asked if and how much they would pay for GSA-Online plus with self-constructed items.

All questionnaires were assessed with the Web-based survey platform, SoSci Survey [48], except the weekly assessments that have been directly implemented in the platform of GSA-Online plus.

\section{Statistical Analyses}

All statistical analyses were done with IBM SPSS Statistics 23 [49]. Recommendation rates and utilization of GSA-Online plus were analyzed with cross-sectional analyses and participants of the Web-based aftercare were compared with the population of all rehabilitants treated during the recruitment period, using descriptive statistics (chi-square tests and $t$ tests, Mann-Whitney 
$\mathrm{U}$ tests if the required assumptions for parametric testing, for example, homogeneity of variance, were violated). Pre-post changes were analyzed with per protocol data as secondary outcomes with longitudinal data analysis ( $t$ tests, rmANOVA, and descriptive statistics). To estimate treatment effects, Cohen $d$ was calculated for the comparison of mean scores with $t$ tests, Cramér V for chi-square tests and the effect size $r$ was calculated for the comparison of median scores with the Mann-Whitney U test.

For the weekly assessment of the general health status and the subjectively rated ability to work, missing data were replaced by the last observation carried forward (LOCF) procedure. To analyze improvement across time, a repeated measures analysis of variance was conducted.

\section{Ethics Approval}

The study protocol was approved by the Ethics Committee of the Federal State of the Rhineland Palatinate (Approval Number: 837.175.16(10494)).

\section{Results}

\section{Recommendation Rates for GSA-Online plus}

Of the 2562 rehabilitants in 2 rehabilitation centers, $112(4.4 \%)$ got a recommendation for GSA-Online plus during inpatient rehabilitation, which was significantly lower than the referral rate to other face-to face aftercare interventions (Table 1). Recommendation rates were higher in psychosomatic rehabilitation, where psychological treatments were routinely recommended in $88 \%(1030 / 1147)$ versus $8 \%(117 / 1147)$ after orthopedic rehabilitation. Rehabilitation aftercare was more frequently prescribed in orthopedic $(500 / 1389,36 \%)$ than in psychosomatic rehabilitation $(89 / 1172,8 \%)$. A total of $11 \%$ $(291 / 2561)$ received recommendations of vocational rehabilitation.

Table 1. Recommendation rates at discharge for aftercare in total and in the 2 rehabilitation centers.

\begin{tabular}{llllllll}
\hline Aftercare recommendation & $\begin{array}{l}\text { Psychosomatic rehabilitation } \\
\text { center }(\mathrm{n}=1172), \mathrm{n}(\%)\end{array}$ & $\begin{array}{l}\text { Orthopedic and rheumatoid } \\
\text { rehabilitation center } \\
(\mathrm{n}=1389), \mathrm{n}(\%)\end{array}$ & $\begin{array}{l}\text { Total }(\mathrm{N}=2561), \\
\mathrm{n}(\%)\end{array}$ & $\chi^{2}{ }_{1}$ & $P$ value & $d$ & Cramér V \\
\hline GSA-Online plus & $69(5.9)$ & $43(3.1)$ & $112(4.4)$ & 11.845 & .001 & 0.14 & -0.068 \\
Psychological Treatment & $1030(87.9)$ & $117(8.4)$ & $1147(44.8)$ & 1623.45 & $<.001$ & 2.63 & -0.796 \\
Rehabilitation aftercare & $89(7.6)$ & $500(36.0)$ & $589(23.0)$ & 289.57 & $<.001$ & 0.71 & 0.336 \\
Vocational rehabilitation & $123(10.5)$ & $168(12.1)$ & $291(11.4)$ & 1.616 & .20 & 0.05 & 0.025 \\
\hline
\end{tabular}

\section{Patients' Characteristics Associated With GSA-Online plus Recommendation}

Rehabilitants who were recommended GSA-Online plus were younger (mean 47.67 [SD 9.97] vs mean 50.31 [SD 9.5], $\left.t_{2559}=2.865, P=.004, d=0.28\right)$ and had longer rehabilitation treatments (median 35 days vs median 28 days, $U=182642.00$, $P<.001, r=-0.12)$. Furthermore, they reported longer work disability before inpatient rehabilitation $(P=.004)$, and were more often (marginally significant, $P=.07$ ) considered able to work at discharge than rehabilitants who did not receive a recommendation (Table 2).

Of the 112 rehabilitants with a recommendation for GSA-Online plus, $77 \%$ (86/112) gave written informed consent and registered on the Web to participate in GSA-Online plus. From these, $58.1 \%(50 / 86)$ wrote at least 6 (completers) and $41.9 \%(36 / 86)$ less than 6 diary entries (dropouts). Multimedia Appendix 5 shows the distribution of the number of diary entries. Dropouts wrote an average of almost 3 diary entries (mean 1.53, [SD 1.72]), completers wrote 10 diary entries (mean 10.34 [SD 2.00]; $\left.t_{84}=-21.4, P<.001, d=-4.68\right)$. Two-thirds of completers $(66.0 \%)$ wrote 11 or 12 diary entries.

Completers were older (mean 49.36 [SD 9.04] vs mean 45.14 [SD 10.49], $t_{84}=-1.998, P=.049, d=0.44$ ) and more often female (74.5\% vs $\left.50 \%, \chi_{1}^{2}=4.638, P=.003\right)$ than dropouts. Further sociodemographic variables revealed no significant differences between completers and dropouts. 
Table 2. Sociodemographic characteristics (T1: baseline): comparison of rehabilitants with and without recommendation for GSA-Online plus.

\begin{tabular}{|c|c|c|c|}
\hline Sociodemographic characteristics & $\begin{array}{l}\text { With recommendation } \\
(\mathrm{n}=112), \mathrm{n}(\%)\end{array}$ & $\begin{array}{l}\text { Without recommendation } \\
(\mathrm{n}=2449), \mathrm{n}(\%)\end{array}$ & Total $(\mathrm{N}=2561), \mathrm{n}(\%)$ \\
\hline \multicolumn{4}{|l|}{$\operatorname{Sex}^{\mathbf{a}}$} \\
\hline Male & $45(40.2)$ & 1067 (43.6) & $1112(43.4)$ \\
\hline Female & $67(59.8)$ & $1382(56.4)$ & 1449 (56.6) \\
\hline \multicolumn{4}{|l|}{ Marital status $^{\mathbf{b}}$} \\
\hline Partnership & $60(53.6)$ & $1525(62.3)$ & $1585(61.9)$ \\
\hline No partnership & $47(42.0)$ & $768(31.4)$ & $815(31.8)$ \\
\hline Unknown & $5(4.5)$ & $156(6.4)$ & $161(6.3)$ \\
\hline \multicolumn{4}{|c|}{ Inability to work before inpatient admission ${ }^{c}$} \\
\hline No inability or not employed & $11(9.8)$ & $347(14.2)$ & $358(14.0)$ \\
\hline 3 months or less & $52(46.4)$ & $1061(43.3)$ & $1113(43.5)$ \\
\hline 3 to 6 months & $29(25.9)$ & $370(15.1)$ & $399(15.6)$ \\
\hline 6 months or more & $20(17.9)$ & $671(27.4)$ & $691(27.0)$ \\
\hline \multicolumn{4}{|l|}{ Work ability at discharge $^{d}$} \\
\hline Able to work & $67(59.8)$ & $1203(49.1)$ & $1270(49.6)$ \\
\hline Unable to work & $44(39.3)$ & $1196(48.8)$ & $1240(48.4)$ \\
\hline Unknown & $1(0.9)$ & $50(2.0)$ & $51(2.0)$ \\
\hline
\end{tabular}

${ }^{\mathrm{a}} \chi_{1}^{2}=0.501, P=.50$, Cramér $V=0.014$.

${ }^{\mathrm{b}} \chi_{2}^{2}=5.713, P=.06$, Cramérs $V=0.047$.

${ }^{c} \chi_{3}^{2}=13.295, P=.004$, Cramérs $V=0.072$.

$\mathrm{d}_{\chi 2}{ }_{2}=5.20, P=.07$, Cramérs $V=0.045$.

\section{Effectiveness of GSA-Online plus and Participants' Satisfaction}

In Figure 1 the weekly ratings of work ability and subjective health status are displayed. Missing data were replaced with the last observation carried forward method.

A statistically significant improvement could be observed for the subjective rated work ability assessed with the WAS $\left(F_{11,759}=3.808, P<.001\right)$ but not for the subjective health status assessed with 1 item of the EQ-5D $\left(F_{11,759}=3.928, P=.22\right)$.

Satisfaction with GSA-Online plus was generally high. $43.5 \%$ (20/46) were largely and $45.7 \%$ (21/46) were very satisfied with GSA-Online plus overall and the level of support was also rated very positive. In total, $47.8 \%$ (22/46) were largely and $32.6 \%$ $(15 / 46)$ were very satisfied with the help they got.

As displayed in Multimedia Appendix 6, for the weekly monitoring of satisfaction and rated helpfulness of the therapeutic feedback, a repeated measures analysis of variance

was calculated. A statistically significant improvement (Multimedia Appendix 6) could be observed for the satisfaction with the feedback from a Web-based therapist $\left(F_{11,176}=2.005\right.$, $P=.03$ ) as well as for the subjectively rated helpfulness $\left(F_{11,176}=2.919, P=.001\right)$ - both assessed weekly with 1 item on a Likert scale from 0 to 4 . For the purpose of sensitivity analysis, data of participants $(\mathrm{N}=17)$ who wrote 12 diary entries were analyzed separately leading to a clear increase of satisfaction and helpfulness (Multimedia Appendix 7).

Table 3 shows secondary outcome measures for regular users of GSA-Online plus (completers). Statistically significant improvements could be observed between discharge from the rehabilitation center and the end of the aftercare program (ie, 3 months after discharge) for subjective work ability as assessed by the WAI $(P=.02, d=0.41)$, perceived stress assessed with the PSS-4 scale $(P=.01, d=-0.38)$, functioning assessed with the SDS $(P=.002, d=-0.60)$, and life satisfaction $(P=.008, d=0.42)$. The other symptom scales scores remained stable and did not change between discharge and the follow-up assessment. 
Figure 1. Means of the subjective health status and subjective work ability, assessed in the course of the aftercare from T2 to T13. Subjective health status was assessed with the EuroQoL-5D single item "How good or bad is your health status today?" $0=$ worst imaginable health; $10=$ =best imaginable health. Subjective work ability was assessed with the Work Ability Score "Current ability to work in comparison with the best, ever reached ability to work"; (range 0-10). N=70, missing data replaced with last observation carried forward.

7

6,5

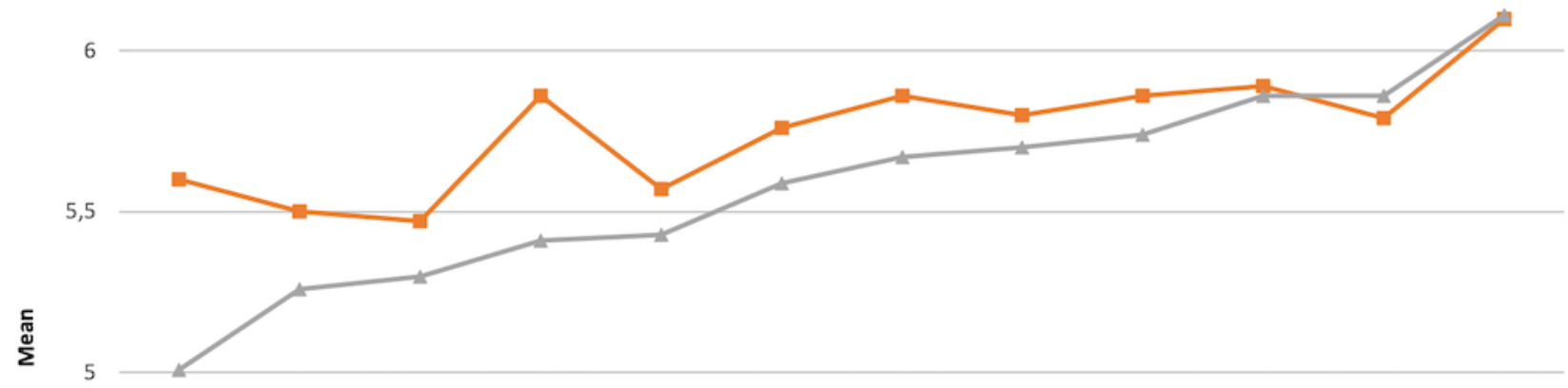

4,5

- -Health status $\quad-$ Work ability

$12 \quad 3$

$\begin{array}{lll}5 & 6 & 7\end{array}$

8

9

10

11

12 
Table 3. Secondary outcome measures of completers of GSA-Online plus at discharge from inpatient medical rehabilitation (T1) and at the end of aftercare (T14).

\begin{tabular}{|c|c|c|c|c|}
\hline Secondary outcomes & $\mathrm{T} 1^{\mathrm{a}}$, mean $(\mathrm{SD})$ & $\mathrm{T} 14^{\mathrm{b}}$, mean $(\mathrm{SD})$ & $P$ value ${ }^{\mathrm{c}}$ & Effect size $(d)^{\mathrm{d}}$ \\
\hline Prognosis of gainful employment $(\mathrm{n}=44)$ & $0.909(1.007)$ & $0.932(0.998)$ & .82 & 0.03 \\
\hline Work ability $(\mathrm{n}=44)$ & $25.080(5.968)$ & $27.125(7.030)$ & $.02^{\mathrm{e}}$ & 0.41 \\
\hline Depression $(n=46)$ & $9.565(4.420)$ & $8.870(4.400)$ & .17 & -0.21 \\
\hline Anxiety $(\mathrm{n}=46)$ & $7.348(4.132)$ & $7.587(4.203)$ & .66 & 0.07 \\
\hline Somatic symptom $(n=45)$ & $11.667(6.142)$ & $11.733(5.618)$ & .90 & 0.02 \\
\hline Psychosocial stress $(\mathrm{n}=46)$ & $7.396(3.655)$ & $7.913(3.601)$ & .26 & 0.17 \\
\hline Perceived stress $(n=44)$ & $7.955(1.976)$ & $7.136(2.339)$ & $.01^{\mathrm{e}}$ & -0.38 \\
\hline General functioning $(\mathrm{n}=44)$ & $22.386(5.406)$ & $18.318(7.398)$ & $.002^{\mathrm{f}}$ & -0.60 \\
\hline Loneliness $(\mathrm{n}=44)$ & $7.727(3.022)$ & $7.144(3.166)$ & .46 & -0.27 \\
\hline Social support $(\mathrm{n}=44)$ & $9.272(2.386)$ & $9.386(2.315)$ & .60 & 0.08 \\
\hline Coping $(n=43)$ & $14.047(3.280)$ & $14.907(3.069)$ & .09 & 0.16 \\
\hline Life satisfaction $(\mathrm{n}=43)$ & $25.581(5.261)$ & $26.814(5.193)$ & $.008^{\mathrm{f}}$ & 0.42 \\
\hline
\end{tabular}

${ }^{\mathrm{a}}$ Baseline.

${ }^{\mathrm{b}}$ End of intervention.

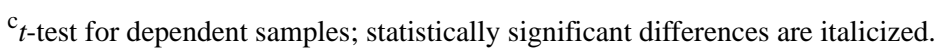

$\mathrm{d} d$ : Cohen $d$.

${ }^{\mathrm{e}}$ Significant at $P<.05$.

f Significant at $P<.01$.

Of the 46 participants who completed the Web-based questionnaire at measurement T14, the majority $(27 / 46,58.7 \%)$ watched at least one of the educational video clips but also almost half $(19 / 46,41.3 \%)$ had not seen any of the films. The main reasons for not watching any films were that the existence of the films was unknown to the participants $(8 / 19,42 \%)$, participants did not use any other features of GSA-Online plus besides the Web-based diary $(5 / 19,26 \%)$ or reported a lack of time $(5 / 19,26 \%)$. In total, $77 \%$ (23/30) of the participants who answered questions about video use assessed the films as a positive contribution to the comprehensibility of GSA-Online plus.

Finally, the willingness of participants to pay for GSA Online Plus was assessed at T14. Participants were asked if they would be willing to pay for GSA-Online plus and how much they would pay if they were required to pay. Of the 46 participants who answered the first question, $37 \%$ (17/46) said they were willing to pay for GSA-Online plus and 40 participants reported to pay in average (mean) 174.25 Euro (SD 292.2, Min 0, Max 1500).

\section{Discussion}

\section{Principal Findings}

Inpatient medical rehabilitation serves to promote work ability and vocational reintegration is a crucial outcome. Although previous Web-based trials have improved coping with work-related stress [23] or showed that Web-based aftercare could successfully maintain effects of inpatient treatment [50], information on implementation processes are missing because previous Web-based interventions have been conducted under study conditions, and participants were usually recruited on the Web. The purpose of the trial was to evaluate the implementation of a Web-based aftercare program (GSA-Online plus) during inpatient medical rehabilitation.

As we had hypothesized, it was feasible to implement the program, and the great majority who received the recommendation $(86 / 112,77 \%)$ actually logged into the program, slightly exceeding our expectations. Among those who started writing blogs, subjective or perceived rated work ability increased over the course of their participation. Satisfaction with Web-based aftercare was generally high. Acceptance was good with $43.5 \%$ (20/46) of the participants being largely and $45.7 \%$ (21/46) very satisfied with GSA-Online plus overall and the level of support was also rated very positively with $47.8 \%(22 / 46)$ of the participants being largely and $32.6 \%(15 / 46)$ very satisfied with the help they received. Contrary to our hypotheses, subjective health status did not increase significantly. However, there was a significant increase of subjective work ability, general functioning, as well as life satisfaction and a decrease of subjective stress. Especially, work ability and functioning are major issues in rehabilitation; therefore, a stabilization of these factors is a good indicator that our aftercare intervention had a focus on the right topics. Unfortunately, no change was found regarding mental distress for the completers of the intervention with a per protocol analysis. An explanation could be, that mental distress was not so high in this sample, with not only psychiatric but also orthopedic main diagnoses, therefore, an improvement was hard to detect in the relatively small sample we could include in our pre-post analyses. 
However, unexpectedly, the referral rate by the physicians of about $4 \%$ was substantially lower than all face-to-face aftercare offers and there was also a lot of individual variation among physicians. Recommendations were made more frequently in psychosomatic (5.9\% [69/1172]) than orthopedic rehabilitation (3.1\% [43/1389]) and more often to younger patients with extended sick leaves and lengthy inpatient stays. This finding coincides with the results of Hennemann et al [51], showing clear preconceptions and barriers regarding Web-based contact among rehabilitation staff, concerning disruption of face-to-face therapeutic alliance and a lack of sufficient data security in Web-based aftercare interventions.

Owing to the low proportion of recommendations, we asked members of the treatment team of the rehabilitation centers in a Web-based survey at the end of the study for their subjective criteria and frequencies of recommendation of GSA-Online plus. Unfortunately, only a few members $(\mathrm{N}=19)$ of the treatment team took part in this assessment and the biggest part (8/19, $61.5 \%$ ) made less than 10 referrals for GSA-Online plus, whereas $23.1 \%(3 / 19)$ referred 11 to 20 and $15.4 \%(2 / 19)$ more than 20 rehabilitants. Members of the treatment team were informed about GSA-Online plus in a one-time on-site training and an informational paper that was accessible to all throughout the study. One explanation for a different referral rate between different team members could well be that especially those who did not attend the training did not make so many recommendations. Data we have on this point at least suggest that there was a trend that referral rate of those who did not attend the training was somehow lower than of those who attended the training. Asked, whether Web-based interventions could be a useful supplement or substitute for regular face-to-face interventions the majority $(8 / 19,42.1 \%)$ answered that they could be a useful supplement but no one rated them as a substitute to usual care.

Our finding is of special interest, because the 121st German congress of physicians has recently lifted the ban on remote treatment [52]. Furthermore, the German statutory pension insurance scheme recently developed requirements for the implementation of tele medical aftercare programs into routine care [53].

Previously, reservations towards occupational e-mental health interventions were also found in patients, especially in the risk groups the interventions were planned for [54]. In spite of the low recommendation rate, the intervention has shown promise in participants. Thus, physicians are in a prime position to provide access to and motivate their patients for sustained participation in these programs. Our findings implicate that 2 steps should be pursued in the future: (1) Awareness about the benefits of Web-based interventions in routine care and their compatibility as an adjunct to face-to face aftercare are required to promote openness in the treatment team to recommend, and to increase referral rates for innovative interventions. (2) Physicians need to be instructed about their patients' reservations to sign up for Web-based support and enabled to help their patients initiate and maintain their engagement in suitable Web-based programs.

\section{Limitations}

Limitations of our study were that we had no control group to analyze efficacy in a routine care setting and that we did not reach our anticipated sample size, because of the much lower referral rate than expected. It also may be debated if logging in once is a sufficient criterion for participation.

The LOCF method for imputing missing data is not the gold standard but seemed sufficient in this small sample, especially as efficacy only was a secondary research question. Furthermore, the multiple comparisons within our secondary research questions are to be considered as a limitation. After adjustment for multiple testing, only general functioning will remain as a significant improvement after participating in GSA-Online plus.

Unfortunately, we did not ask participants about advantages of Web-based interventions but we asked them about their digital competencies. Two-thirds of the participants rated their knowledge of using digital media (eg, PC, smartphone, and tablet) as mediocre to rather good. Overall, the attitude toward the internet was positive, but one-third also stated that they feel burdened by the constant availability via mobile phone or email.

Hence, future implementations should focus more on collaborating with staff and clinicians in rehabilitation clinics to address potential prejudices and barriers to Web-based aftercare. On the patient side, it is important to address advantages and disadvantages of Web-based interventions to improve acceptance for internet- and mobile-based interventions.

\section{Conclusions}

Web-based psychological aftercare proved to be effective to reduce treatment gaps after inpatient medical rehabilitation but only for a limited number of rehabilitants. GSA-Online plus has been provided as a Web-based aftercare that offers all inpatient medical rehabilitation patients with occupational exposure, mental comorbidity, and an intentional timely return to work the opportunity to be promoted and assisted with professional reintegration by trained psychologists. Once a recommendation for GSA-Online plus was given from the physician in routine care, it could lead to a significantly higher participant motivation and adherence than in a controlled efficacy study. Outcome criteria on which the Web-based aftercare focuses (ability to function, work ability, general state of health, and life satisfaction) will improve even further in the course of follow-up, compared with the state of health at the end of inpatient rehabilitation. Motivation of rehabilitants and attitudes of the treatment team toward Web-based interventions are essential to improve implementation/recommendation rates. An important question for future research could be how Web-based interventions for rehabilitants with work-related problems could be optimized or supplemented to reach more rehabilitants. As the return to work is a major issue in rehabilitative treatment in Germany, a more practical oriented intervention with a more social work-driven focus could possibly close this gap and continue the multidisciplinary treatment approach that is already one main characteristic of inpatient medical rehabilitation in Germany. 


\section{Acknowledgments}

The authors would like to express their gratitude to all participants of the trial as well as the research assistants of the Mittelrhein-Klinik in Boppard/Bad Salzig, Germany (Melanie Mayeh, Dietmar Kraeber), the Karl Aschoff Klinik in Bad Kreuznach, Germany (Anja Zocher-Tiegs) and the Drei-Burgen-Klinik, Bad Kreuznach (Michael Wiegert, Vera Simon-Reichert). This study was funded by the German Statutory Pension Insurance Scheme (Deutsche Rentenversicherung Bund; grant number: 0423/40-64-50-50) from 08/2015 to $10 / 2017$.

\section{Conflicts of Interest}

None declared.

\section{Multimedia Appendix 1}

Screenshot of the landing page.

[PDF File (Adobe PDF File), 690KB-Multimedia Appendix 1]

\section{Multimedia Appendix 2}

Overview of the online diary.

[PDF File (Adobe PDF File), 203KB-Multimedia Appendix 2]

\section{Multimedia Appendix 3}

Screenshot of an explanatory video.

[PDF File (Adobe PDF File), 1MB-Multimedia Appendix 3]

\section{Multimedia Appendix 4}

Screenshot of an explanatory video.

[PDF File (Adobe PDF File), 1MB-Multimedia Appendix 4]

\section{Multimedia Appendix 5}

Number of written online diary entries (completer vs dropouts).

[PDF File (Adobe PDF File), 34KB-Multimedia Appendix 5]

\section{Multimedia Appendix 6}

Means of the weekly monitoring of satisfaction and rated helpfulness of the therapeutic feedback, both assessed weekly with one item each on a Likert-scale from 0 to 4 . $\mathrm{N}=70$, missing data replaced with last observation carried forward.

[PDF File (Adobe PDF File), 58KB-Multimedia Appendix 6]

\section{Multimedia Appendix 7}

Means of the weekly monitoring of satisfaction and rated helpfulness of the therapeutic feedback, both assessed weekly with one item each on a Likert-scale from 0 to 4 . $\mathrm{N}=17$, per protocol data.

[PDF File (Adobe PDF File), 62KB-Multimedia Appendix 7]

\section{References}

1. Bethge M, Radoschewski FM, Müller-Fahrnow W. Work stress and work ability: cross-sectional findings from the German sociomedical panel of employees. Disabil Rehabil 2009 Sep;31(20):1692-1699. [doi: 10.1080/09638280902751949] [Medline: 19479539]

2. Stansfeld S, Candy B. Psychosocial work environment and mental health - a meta-analytic review. Scand J Work Environ Health 2006 Dec;32(6):443-462. [doi: 10.5271/sjweh.1050]

3. Henderson M, Harvey SB, Overland S, Mykletun A, Hotopf M. Work and common psychiatric disorders. J R Soc Med 2011 May;104(5):198-207 [FREE Full text] [doi: 10.1258/jrsm.2011.100231] [Medline: 21558098] 
4. [German Pension Insurance]. Berlin: Deutsche Rentenversicherung Bund; 2014. [Position paper of the Deutsche Rentenversicherung on the importance of mental illness in rehabilitation and reduction of earning capacity] URL: https:/ /tinyurl.com/y2uvz9ql [accessed 2019-06-04]

5. Bethge M. [Work-related medical rehabilitation]. Rehabilitation (Stuttg) 2017 Feb;56(1):14-21. [doi: 10.1055/s-0042-118579] [Medline: 28219096]

6. Briest J, Bethge M. [Intensified work-related rehabilitation aftercare: long-term results of a randomized controlled multicenter trial]. Rehabilitation (Stuttg) 2016 Apr;55(2):108-114. [doi: 10.1055/s-0042-102998] [Medline: 27070985]

7. Bethge M. Effects of graded return-to-work: a propensity-score-matched analysis. Scand J Work Environ Health 2016 Dec 1;42(4):273-279 [FREE Full text] [doi: 10.5271/sjweh.3562] [Medline: 27074050]

8. Kobelt A, Nickel L, Grosch E, Lamprecht F, Künsebeck HW. [Participation in psychosomatic outpatient care after in-patient rehabilitation]. Psychother Psychosom Med Psychol 2004 Feb;54(2):58-64. [doi: 10.1055/s-2003-812612] [Medline: 14872392]

9. Sibold M, Mittag O, Kulick B, Müller E, Opitz U, Jäckel WH. [Predictors of participation in medical rehabilitation follow-up in working patients with chronic back pain]. Rehabilitation (Stuttg) 2011 Dec;50(6):363-371. [doi: 10.1055/s-0031-1271815] [Medline: 21647850]

10. Richards D, Richardson T. Computer-based psychological treatments for depression: a systematic review and meta-analysis. Clin Psychol Rev 2012 Jun;32(4):329-342. [doi: 10.1016/j.cpr.2012.02.004] [Medline: 22466510]

11. Andrews G, Cuijpers P, Craske MG, McEvoy P, Titov N. Computer therapy for the anxiety and depressive disorders is effective, acceptable and practical health care: a meta-analysis. PLoS One 2010;5(10):e13196 [FREE Full text] [doi: 10.1371/journal.pone.0013196] [Medline: 20967242]

12. Macea DD, Gajos K, Daglia CY, Fregni F. The efficacy of web-based cognitive behavioral interventions for chronic pain: a systematic review and meta-analysis. J Pain 2010 Oct;11(10):917-929. [doi: 10.1016/j.jpain.2010.06.005] [Medline: 20650691]

13. Rooke S, Thorsteinsson E, Karpin A, Copeland J, Allsop D. Computer-delivered interventions for alcohol and tobacco use: a meta-analysis. Addiction 2010 Aug;105(8):1381-1390. [doi: 10.1111/j.1360-0443.2010.02975.x] [Medline: 20528806]

14. Davies C, Spence J, Vandelanotte C, Caperchione C, Mummery W. Meta-analysis of internet-delivered interventions to increase physical activity levels. Int J Behav Nutr Phys Act 2012 Apr 30;9:52 [FREE Full text] [doi: 10.1186/1479-5868-9-52] [Medline: 22546283]

15. Grunenberg E, Lin J, Baumeister H. [Effectiveness of web-based psychological interventions for weight loss--a systematic review]. Rehabilitation (Stuttg) 2013 Jun;52(3):182-187. [doi: 10.1055/s-0033-1343489] [Medline: 23761207]

16. Kanera IM, Willems RA, Bolman CA, Mesters I, Zambon V, Gijsen BC, et al. Use and appreciation of a tailored self-management eHealth intervention for early cancer survivors: process evaluation of a randomized controlled trial. $\mathbf{J}$ Med Internet Res 2016;18(8):e229 [FREE Full text] [doi: 10.2196/jmir.5975] [Medline: 27554525]

17. Koch W, Frees B. [ARD-ZDF Online Study | ARD / ZDF Media Commission]. ARD/ZDF-Onlinestudie 2017: Neun von zehn Deutschen online. Media perspektiven. 2017; 2017. [ARD/ZDF online study 2017: Nine out of ten Germans online] URL: http://www.ard-zdf-onlinestudie.de/files/2017/Artikel/917 Koch Frees.pdf [accessed 2019-06-06] [WebCite Cache ID 78vQqmO5m]

18. Eichenberg C, Wolters C, Brähler E. The internet as a mental health advisor in Germany--results of a national survey. PLoS One 2013;8(11):e79206 [FREE Full text] [doi: 10.1371/journal.pone.0079206] [Medline: 24278121]

19. Baumeister H, Lin J, Ebert DD. [Internet- and mobile-based approaches : psycho-social diagnostics and treatment in medical rehabilitation]. Bundesgesundheitsblatt Gesundheitsforschung Gesundheitsschutz 2017 Apr;60(4):436-444. [doi: 10.1007/s00103-017-2518-9] [Medline: 28224187]

20. Donker T, Blankers M, Hedman E, Ljótsson B, Petrie K, Christensen H. Economic evaluations of Internet interventions for mental health: a systematic review. Psychol Med 2015 Dec;45(16):3357-3376. [doi: 10.1017/S0033291715001427] [Medline: 26235445]

21. Heber E, Ebert DD, Lehr D, Cuijpers P, Berking M, Nobis S, et al. The benefit of web-and computer-based interventions for stress: a systematic review and meta-analysis. J Med Internet Res 2017 Feb 17;19(2):e32 [FREE Full text] [doi: 10.2196/jmir.5774] [Medline: 28213341]

22. Ebert DD, Heber E, Berking M, Riper H, Cuijpers P, Funk B, et al. Self-guided internet-based and mobile-based stress management for employees: results of a randomised controlled trial. Occup Environ Med 2016 May;73(5):315-323. [doi: 10.1136/oemed-2015-103269] [Medline: 26884049]

23. Heber E, Lehr D, Ebert DD, Berking M, Riper H. Web-based and mobile stress management intervention for employees: a randomized controlled trial. J Med Internet Res 2016;18(1):e21 [FREE Full text] [doi: 10.2196/jmir.5112] [Medline: 26818683]

24. Eichenberg C, Ott R. [Clinical-psychological intervention on the internet]. Psychotherapeut 2011 Jun 10;57(1):58-69 [FREE Full text] [doi: 10.1007/s00278-011-0832-5]

25. Zwerenz R, Becker J, Gerzymisch K, Siepmann M, Holme M, Kiwus U, et al. Evaluation of a transdiagnostic psychodynamic online intervention to support return to work: A randomized controlled trial. PLoS One 2017;12(5):e0176513 [FREE Full text] [doi: 10.1371/journal.pone.0176513] [Medline: 28481893] 
26. Linden M. Psychosomatic inpatient rehabilitation: the German model. Psychother Psychosom 2014;83(4):205-212. [doi: 10.1159/000358852] [Medline: 24970244]

27. Zwerenz R, Böhme K, Wirth A, Labitzke N, Pachtchenko S, Beutel ME. Integration of the work-related online aftercare intervention 'GSA-online plus' (healthy and without stress at the workplace) into clinical practice: study protocol for an implementation study. BMC Health Serv Res 2018 Dec 2;18(1):312 [FREE Full text] [doi: 10.1186/s12913-018-2995-z] [Medline: 29716605]

28. Beutel M, Böhme K, Banerjee M, Zwerenz R. Psychodynamic online treatment following supportive expressive therapy (SET): therapeutic rationale, interventions and treatment process. Z Psychosom Med Psychother 2018 Jun;64(2):186-197. [doi: 10.13109/zptm.2018.64.2.186] [Medline: 29862925]

29. Matomo: \#1 Secure Open Web Analytics Platform. URL: https://matomo.org [accessed 2019-06-04] [WebCite Cache ID 78vYkRjXk]

30. Mittag O, Raspe H. [A brief scale for measuring subjective prognosis of gainful employment: findings of a study of 4279 statutory pension insurees concerning reliability (Guttman scaling) and validity of the scale]. Rehabilitation (Stuttg) 2003 Jun;42(3):169-174. [doi: 10.1055/s-2003-40095] [Medline: 12813654]

31. Ilmarinen J. The Work Ability Index (WAI). Occup Med (Lond) 2006 Oct 17;57(2):160. [doi: 10.1093/occmed/kqm008]

32. Löwe B, Spitzer R, Zipfel S, Herzog W. [University Hospital Heidelberg - University of Heidelberg]. Karlsruhe: Pfizer; 2002. [PHQ-D: Health questionnaire for patients] URL: https://www.klinikum.uni-heidelberg.de/fileadmin/ Psychosomatische Klinik/download/PHQ Manual1.pdf [accessed 2019-06-04] [WebCite Cache ID 78s9N4k7g]

33. Kroenke K, Spitzer RL, Williams JB. The PHQ-9: validity of a brief depression severity measure. J Gen Intern Med 2001 Sep;16(9):606-613 [FREE Full text] [Medline: 11556941]

34. Gräfe K, Zipfel S, Herzog W, Löwe B. [Screening of mental disorders with the "Health Questionnaire for Patients (PHQ-D)"]. Diagnostica 2004 Oct;50(4):171-181. [doi: 10.1026/0012-1924.50.4.171]

35. Klapow J, Kroenke K, Horton T, Schmidt S, Spitzer RB, Williams JB. Psychological disorders and distress in older primary care patients: a comparison of older and younger samples. Psychosom Med 2002;64(4):635-643. [doi: 10.1097/01.psy.0000021942.35402.c3] [Medline: 12140354]

36. Spitzer RL, Kroenke K, Williams JB, Löwe B. A brief measure for assessing generalized anxiety disorder: the GAD-7. Arch Intern Med 2006 May 22;166(10):1092-1097. [doi: 10.1001/archinte.166.10.1092] [Medline: 16717171]

37. Gierk B, Kohlmann S, Kroenke K, Spangenberg L, Zenger M, Brähler E, et al. The somatic symptom scale-8 (SSS-8): a brief measure of somatic symptom burden. JAMA Intern Med 2014 Mar;174(3):399-407. [doi:

10.1001/jamainternmed.2013.12179] [Medline: 24276929]

38. Cohen S, Williamson G. Perceived stress in a probability sample of the United States. In: Spacapan S, Oskamp S, editors. The Social Psychology of Health: The Claremont Symposium on Applied Social Psychology. Newbury Park, CA: Sage Publications; 1988:31-67.

39. Sheehan DV, Harnett-Sheehan K, Raj BA. The measurement of disability. Int Clin Psychopharmacol 1996 Jun;11(Suppl 3):89-95. [Medline: $\underline{8923116]}$

40. Leon AC, Olfson M, Portera L, Farber L, Sheehan DV. Assessing psychiatric impairment in primary care with the Sheehan Disability Scale. Int J Psychiatry Med 1997;27(2):93-105. [doi: 10.2190/T8EM-C8YH-373N-1UWD] [Medline: 9565717]

41. Dalgard OS. Community mental health profile as tool for psychiatric prevention. In: Trent DR, Reed C, editors. Promotion of Mental Health, 1995, Volume 5. Avebury: Ashgate Publishing, Limited; 1996.

42. Kocalevent R, Zenger M, Hinz A, Klapp B, Brähler E. Resilient coping in the general population: standardization of the brief resilient coping scale (BRCS). Health Qual Life Outcomes 2017 Dec 28;15(1):251 [FREE Full text] [doi: 10.1186/s12955-017-0822-6] [Medline: 29282066]

43. Richter D, Weinhardt M. LS-S: Loneliness Scale-SOEP. In: Kemper CJ, Zenger M, Brähler E, editors. [Psychological and Social Sciences Short Scales: Standardized Survey Instruments for Science and Practice]. Berlin: MWV Medizinisch Wissenschaftliche Verlagsgesellschaft; 2014:192-193.

44. Henrich G, Herschbach P. Questions on Life Satisfaction (FLZM): a short questionnaire for assessing subjective quality of life. Eur J Psychol Assess 2000 Sep;16(3):150-159. [doi: 10.1027//1015-5759.16.3.150]

45. Schmidt J, Lamprecht F, Wittmann WW. [Satisfaction with inpatient care: development of a questionnaire and first validity assessments]. Psychother Med Psychol 1989;39(7):248-255.

46. Graf J, Claes C, Greiner W, Uber A. [The German version of the EuroQol questionnaire]. J Public Health 1998 Mar;6(1):3-20. [doi: $10.1007 / \mathrm{BF} 02956350]$

47. El Fassi M, Bocquet V, Majery N, Lair ML, Couffignal S, Mairiaux P. Work ability assessment in a worker population: comparison and determinants of Work Ability Index and Work Ability score. BMC Public Health 2013 Apr 8;13:305 [FREE Full text] [doi: 10.1186/1471-2458-13-305] [Medline: 23565883]

48. Leiner D. SoSci Survey. SoSci Survey (Version 3.1.06) URL: https://www.soscisurvey.de [accessed 2019-06-04] [WebCite Cache ID 78sB7xiya]

49. IBM Corp. Armonk, NY: IBM Corp; 2014. IBM SPSS Statistics for Windows, Version 23 URL: https://www-01.ibm.com/ support/docview.wss?uid=swg24038592 [accessed 2019-06-06] [WebCite Cache ID 78vU8qlZB] 
50. Ebert D, Tarnowski T, Gollwitzer M, Sieland B, Berking M. A transdiagnostic internet-based maintenance treatment enhances the stability of outcome after inpatient cognitive behavioral therapy: a randomized controlled trial. Psychother Psychosom 2013;82(4):246-256. [doi: 10.1159/000345967] [Medline: 23736751]

51. Hennemann S, Beutel ME, Zwerenz R. Ready for eHealth? Health professionals' acceptance and adoption of eHealth interventions in inpatient routine care. J Health Commun 2017 Mar;22(3):274-284. [doi: 10.1080/10810730.2017.1284286] [Medline: 28248626]

52. Korzilius H. [Opening of the 121th German Medical Conference: wrestling for correct arguments]. Dtsch Arztebl Int 2018;115(19):907-909 [FREE Full text]

53. Deutsche Rentenversicherung Bund. Anforderungen an Tele-Reha-Nachsorge. Berlin: Deutsche Rentenversicherung Bund; 2018. URL: https://tinyurl.com/y6jr4y5b [accessed 2019-06-04]

54. Hennemann S, Witthöft M, Bethge M, Spanier K, Beutel ME, Zwerenz R. Acceptance and barriers to access of occupational e-mental health: cross-sectional findings from a health-risk population of employees. Int Arch Occup Environ Health 2018 Apr;91(3):305-316. [doi: 10.1007/s00420-017-1280-5] [Medline: 29189895]

\author{
Abbreviations \\ EQ-5D: EuroQol questionnaire \\ GSA: Gesund und Stressfrei am Arbeitsplatz [Healthy and stress-less at the workplace] \\ LOCF: Last observation carried forward \\ PHQ: Patient Health Questionnaire \\ PSS-4: Perceived Stress Scale \\ WAI: Work ability index \\ WAS: Work Ability Score \\ WMR: Work-related medical rehabilitation
}

\author{
Edited by G Eysenbach; submitted 02.10.18; peer-reviewed by E Neter, J Lin, C Eichenberg, K Spanier; comments to author 22.11.18; \\ revised version received 20.02.19; accepted 26.04.19; published 18.06.19 \\ Please cite as: \\ Zwerenz R, Baumgarten C, Dahn I, Labitzke N, Schwarting A, Rudolph M, Ferdinand P, Dederichs-Masius U, Beutel ME \\ Implementation of a Web-Based Work-Related Psychological Aftercare Program Into Clinical Routine: Results of a Longitudinal \\ Observational Study \\ J Med Internet Res 2019;21(6):e12285 \\ URL: http://www.jmir.org/2019/6/e12285/ \\ doi: $\underline{10.2196 / 12285}$ \\ PMID: 31215515
}

CRüdiger Zwerenz, Carlotta Baumgarten, Ingo Dahn, Nicole Labitzke, Andreas Schwarting, Matthias Rudolph, Peter Ferdinand, Ute Dederichs-Masius, Manfred E Beutel. Originally published in the Journal of Medical Internet Research (http://www.jmir.org), 18.06.2019. This is an open-access article distributed under the terms of the Creative Commons Attribution License (https://creativecommons.org/licenses/by/4.0/), which permits unrestricted use, distribution, and reproduction in any medium, provided the original work, first published in the Journal of Medical Internet Research, is properly cited. The complete bibliographic information, a link to the original publication on http://www.jmir.org/, as well as this copyright and license information must be included. 